\title{
INVERSE PROBLEM OF A ONE-DIMENSIONAL MODEL IN MULTILAYER HEAT CONDUCTION
}

\author{
G.C. Oliveira ${ }^{\mathrm{a}}$, \\ S.S. Ribeiro ${ }^{\mathrm{a}}$ \\ and G. Guimarães ${ }^{b}$ \\ ${ }^{a}$ State University of Minas Gerais \\ Ituiutaba, Minas Gerais, Brazil. \\ gabriela.oliveira@uemg.br \\ sidney.silveira@uemg.br \\ ${ }^{b}$ Federal University of Uberlândia \\ School of Mechanical Engineering \\ Uberlândia, Minas Gerais, Brazil. \\ gguima@ufu.br \\ ABSTRACT \\ The inverse problem in conducting heat is related to the determination of \\ the boundary condition, rate of heat generation, or thermophysical \\ properties, using temperature measurements at one or more positions of the \\ solid. The inverse problem in conducting heat is mathematically one of the \\ ill-posed problems, because its solution extremely sensitive to measurement \\ errors. For a well-placed problem the following conditions must be \\ satisfied: the solution must exist, it must be unique and must be stable on \\ small changes of the input data. The objective of the work is to estimate the \\ heat flux generated at the tool-chip-chip interface in a manufacturing \\ process. The term "estimation" is used because in the temperature \\ measurements, errors are always present and these affect the accuracy of the \\ calculation of the heat flow. \\ Received: February 19, 2020 \\ Revised: March 04, 2020 \\ Accepted: May 28, 2020 \\ Keywords: inverse problem; Green's functions; heat conduction
}

\section{NOMENCLATURE}

$\mathrm{k}_{\mathrm{i}} \quad$ thermal conductivity in the $i$ direction, $\mathrm{i}=1,2,3, \ldots, \mathrm{W} /(\mathrm{m} \cdot \mathrm{K})$

$\mathrm{L} \quad$ length, $\mathrm{m}$

b layer length, $\mathrm{m}$

q heat flux, $\mathrm{W} / \mathrm{m}^{2}$

$\mathrm{T}$ temperature, ${ }^{\circ} \mathrm{C}$

$\mathrm{t}$ time, $\mathrm{s}$

$\mathrm{N}$ norm

G Green function

$\mathrm{h}$ impulsive response

$\mathrm{H}$ transfer function

$\mathcal{L} \quad$ Laplace Transform

$\mathrm{x}, \mathrm{y}, \mathrm{z}$ cartesian coordinates, $\mathrm{m}$

\section{Greek symbols}

$\alpha \quad$ thermal diffusivity, $\mathrm{m}^{2} / \mathrm{s}$

$\beta \quad$ eigenvalue

$\lambda \quad$ eigenvalue

$\eta \quad$ eigenvalue

$\tau \quad$ delayed time

$\delta \quad$ Dirac delta pseudo fucntion

$\rho \quad$ density, $\mathrm{kg} / \mathrm{m}^{3}$

\section{INTRODUCTION}

In the machining process, the use of inverse problems is common for the estimationof the temperature at which the tip of the cutting tool is located. The direct measurement ofsensor temperature becomes complicated due to the rotating movement of the tool,the use of thermocouples, and the release of chips, which makes the measurement byradiation. The temperature of the cutting tool influences its useful life and abetter control of machining parameters such as feed and cut speeds,enables increased productivity and lower production costs.

Several techniques are used in the inverse problem solutions in heat conduction, such as: least squares method, Duhamel's theorem, specified;function, genetic algorithm, simulated annealing, more recently, has been used filters for solution of heat conduction inverse problems. Another technique has been proposed and developed by(FERNANDES, 2013) that relates inverse problem with transfer function TFBGF (transfer function based on Green's function).

The methodology of the technique (TFBGF) consists in determining the transfer function of a thermal system. With the transfer function it is possible to establish the heat flux estimation method by different approaches, through the deconvolution.

That is, knowing the transfer function $H(x, t)$ and temperatures $T(x, t)$ (experimental or theoretical) it is possible to estimate the heat flux doing: $q(x, s)=$ $T(x, s) H^{-1}(x, s)$, given in the Laplace domain (FERNANDES, 2013). 


\section{THEORY}

\section{Direct problem multilayer}

The thermal problem is defined by a flat plate, with two layers, subjected to a heat flow, $q(t)$ at $x=0$, and thermal insulation condition on the opposite surface, $x=L$, whose thermophysical properties are different in each layer, delimited by $x=b$ Fig. (1), is referenced as $X 2 C 12$ by(HajiSheikh, 2014).

The multilayer analytical, represented by Fig. (1) was developed by(Oliveira, 2015) and it has as analytical solution:

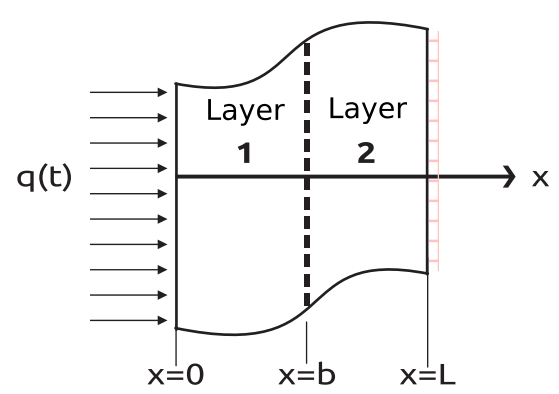

Figure 1. Thermal problem: flat plate, with two layers, subjected to a head flux at $x=0$, and thermal insulation condition on the opposite surface, $x=L$.

It is observed that to obtain the thermal profile of the problem $X 2 C 12$ a particular case is used, where the heat flux imposed at one end $x=0$ is known and is costly, leaving only the calculation of the integrals in the Eqs. (1).

$$
\begin{aligned}
& T_{1}(x, t){ }^{\infty} \\
& =\frac{\alpha_{1}}{k_{1}} \sum_{n=1}^{\infty} \frac{X_{1 n}}{N_{x}} \int_{0}^{t} e^{-\lambda_{n}^{2}(t-\tau)} \int_{x_{1}}^{x_{2}} X_{1 n}\left(x^{\prime}\right) \\
& \times q(t) \delta\left(x^{\prime}-0\right) d x^{\prime} d \tau \\
& T_{1}(x, t)=\frac{\alpha_{1}}{k_{1}} \sum_{n=1}^{\infty} \frac{X_{1 n}(x) X_{1 n}(0)}{N_{x}} \\
& T_{1}(x, t)=\frac{\alpha_{1}}{k_{1}} \sum_{n=1}^{\infty} \frac{\cos (\gamma x) \cos (0)}{N_{x}} q(t) e^{-\lambda_{n}^{2}(t-\tau)} d \tau \\
& \times \int_{0}^{t} q(t) e^{-\lambda_{n}^{2}(t-\tau)} d \tau
\end{aligned}
$$

$T_{2}(x, t)$

$=\frac{\alpha_{1}}{k_{1}} \sum_{n=1}^{\infty} \frac{X_{2 n}}{N_{x}} \int_{0}^{t} e^{-\lambda_{n}^{2}(t-\tau)} \int_{x_{1}}^{x_{2}} X_{1 n}\left(x^{\prime}\right)$
$\times q(t) \delta\left(x^{\prime}-0\right) d x^{\prime} d \tau$

$$
\begin{aligned}
T_{2}(x, t)=\frac{\alpha_{1}}{k_{1}} \sum_{n=1}^{\infty} & \frac{X_{2 n}(x) X_{1 n}(0)}{N_{x}} \\
& \times \int_{0}^{t} q(t) e^{-\lambda_{n}^{2}(t-\tau)} d \tau
\end{aligned}
$$

$T_{2}(x, t)$

$=\frac{\alpha_{1}}{k_{1}} \sum_{n=1}^{\infty} \frac{1}{N_{x}}\{[\cos (\eta b) \cos (\gamma b)$

$\left.+\left(\frac{k_{1}}{k_{2}}\right)\left(\frac{\gamma}{\eta}\right) \operatorname{sen}(\gamma b) \operatorname{sen}(\eta b)\right] \cos (\eta x)$

$+[\cos (\gamma b) \operatorname{sen}(\eta b)$

$\left.\left.-\left(\frac{k_{1}}{k_{2}}\right)\left(\frac{\gamma}{\eta}\right) \operatorname{sen}(\gamma b) \cos (\eta b)\right] \operatorname{sen}(\eta x)\right\} \cos (0)$

$\times \int_{0}^{t} q(t) e^{-\lambda_{n}^{2}(t-\tau)} d \tau$

Where $\lambda_{\mathrm{n}}$ are eigenvalues and $\gamma$ and $\eta$ are related to $\lambda$ by the following expression

$$
\gamma^{2}=\frac{\lambda^{2}}{\alpha_{1}} ; \eta^{2}=\frac{\lambda^{2}}{\alpha_{2}}
$$

For example, if the heat flux is constant or only dependent on the position, or even an exponential function, $q(t)=c_{1} e^{-c_{2} t}$, with $c_{1}$ and $c_{2}$ nonzero, the solution is easily determined analytically. However, in a real situation the heat flux, $q(t)$, is not described by an analytical expression, since its nature is discrete. In this case, the solution could be called a "hybrid", because the integral is necessarily calculated from the heat flow discretization,(FERNANDES, 2013).The hybrid solution that is used in a real situation is shown below, since the heat flux is discrete.

\section{Hybrid solution}

The hybrid solution is an alternative to real cases where the heat flux is not constant. In this case, the experimental heat flux (discrete data) is represented as a vector where each component is a flux value, and this flux is considered constant for each time interval, as shown by Fig (2), that is, $q(t)=\left[q_{1}, q_{2}, q_{3}, \cdots, q_{n}\right]$ being $q_{n}$ the component for the interval $\Delta_{t}=t_{n+1}-t_{n}$ with $n=1,2,1$ Fernandes (2013). Thus, the integral appearing in Eqs. (1- 2) can be expressed by

$$
\begin{gathered}
\int_{0}^{t} q(t) e^{-\lambda_{n}^{2}(t-\tau)} d \tau=\int_{t_{1}=0}^{t_{2}} q_{1} e^{-\lambda_{n}^{2}(t-\tau)} d \tau \\
+\int_{t_{2}}^{t_{3}} q_{2} e^{-\lambda_{n}^{2}(t-\tau)} d \tau+\cdots \\
+\int_{t_{n}}^{t_{n+1}} q_{n} e^{-\lambda_{n}^{2}(t-\tau)} d
\end{gathered}
$$




$$
=\frac{1}{-\lambda_{n}^{2} t} \sum_{n=1}^{N-1} q_{n}\left(e^{-\lambda_{n}^{2}(t-\tau)}-e^{-\lambda_{n}^{2}\left(t_{n}\right)}\right)
$$

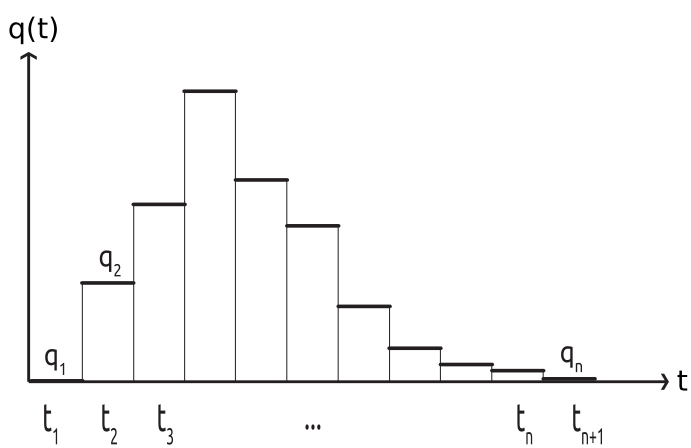

Figure 2. Discrete heat flow.

The analytical solution is obtained by considering the heat flux, $q(t)=c_{1} e^{-c_{2} t}$ in Eq. (1) and thus, solving the integrals in the time of the Eqs. (1a-1b).

$$
=\frac{\alpha_{1}}{k_{1}} \sum_{n=1}^{\infty} \frac{\cos (\gamma x) \cos (0)}{N_{x}} \frac{c_{1}\left(e^{-c_{2} t}-e^{-\lambda_{2} t}\right)}{\lambda_{n}^{2}-c_{2}}
$$

$$
\begin{aligned}
& T_{2}(x, t) \\
& =\frac{\alpha_{1}}{k_{1}} \sum_{n=1}^{\infty} \frac{1}{N_{x}}\{[\cos (\eta b) \cos (\gamma b) \\
& \left.+\left(\frac{k_{1}}{k_{2}}\right)\left(\frac{\gamma}{\eta}\right) \operatorname{sen}(\gamma b) \operatorname{sen}(\eta b)\right] \cos (\eta x) \\
& +[\cos (\gamma b) \operatorname{sen}(\eta b) \\
& \left.\left.-\left(\frac{k_{1}}{k_{2}}\right)\left(\frac{\gamma}{\eta}\right) \operatorname{sen}(\gamma b) \cos (\eta b)\right] \operatorname{sen}(\eta x)\right\} \cos (0) \\
& \times \frac{c_{1}\left(e^{-c_{2} t}-e^{-\lambda_{2} t}\right)}{\lambda_{n}^{2}-c_{2}}
\end{aligned}
$$

The solutions given by Eqs.(1-4) are implemented in MATLAB with the following physical and geometric characteristics, thermal conductivity, $k_{1}=401[\mathrm{~W} / \mathrm{mK}], k_{2} 401[\mathrm{~W} / \mathrm{mK}]$, thermal diffusivity, $\alpha_{1}=117 \times 10^{-6}\left[\mathrm{~m}^{2} / \mathrm{s}\right]$, initial temperature $T_{0}=0\left[{ }^{\circ} \mathrm{C}\right]$, plate length, $L=5 \times$ $10^{-2}[\mathrm{~m}]$ and $d t=0.5$ and $0.01[\mathrm{~s}]$. For the discrete heat flux, applied only in Eq.(4), we have the vector $q_{n}=\left[0, c_{1} \exp \left(-c_{2} t\right)\right]$ with $c_{1}=4 \times 10^{5}$ and $c_{2}=$ 0.002 .

The Tab.(1) shows the comparison between purely analytical Eq.(1) and hybrid solutions Eq.
(4).It is observed that the smaller time discretization interval the closer the solutions will be to each other.

Table 1. Comparison between the solutions obtained by Eqs (1) and (4).

\begin{tabular}{|c|ccc|ll|}
\hline $\mathrm{x}=0$ & \multicolumn{3}{|c|}{$\mathrm{dt}=0.5 \mathrm{~s}$} & \multicolumn{2}{c|}{$\mathrm{dt}=0.01 \mathrm{~s}$} \\
\hline $\mathrm{t}$ & $\mathrm{Eq}(1)$ & $\mathrm{Eq}(5)$ & $\mathrm{dif}$ & $\mathrm{Eq}(5)$ & $\mathrm{dif}$ \\
\hline 0.0 & 0.0000 & 0.0000 & 0.0000 & 0.0000 & 0.0000 \\
1.0 & 4.0033 & 4.0513 & 0.0480 & 4.0039 & 0.0006 \\
2.0 & 6.6592 & 6.7533 & 0.0941 & 6.6593 & 0.0001 \\
3.0 & 9.0282 & 9.1498 & 0.0121 & 9.0323 & 0.0041 \\
4.0 & 11.3479 & 11.4883 & 0.1404 & 11.3533 & 0.0054 \\
5.0 & 13.6561 & 13.8122 & 0.1561 & 13.6626 & 0.0065 \\
6.0 & 15.9585 & 15.1338 & 0.1753 & 15.9661 & 0.0076 \\
7.0 & 18.2561 & 18.4578 & 0.2017 & 18.2649 & 0.0088 \\
8.0 & 20.8376 & 20.7726 & 0.2234 & 20.5591 & 0.0099 \\
9.0 & 22.8376 & 23.0789 & 0.2413 & 22.8487 & 0.0111 \\
10.0 & 24.1213 & 24.3819 & 0.2606 & 24.1103 & 0.0110 \\
& \multicolumn{5}{|c|}{} \\
\hline
\end{tabular}

\section{Transfer Function and Multilayer Inverse Problem}

In dynamic systems there are three variables to be studied, the excitation, the transfer function and the response of the system, in this way the problems are solved knowing always two variables and estimating the third one: the inverse problems are those in which from the knowledge of the system response (effect) the excitation (cause) is estimated. It is possible to analyze heat conduction problems by making an analogy to dynamic systems (Fernandes,2013).

To obtain the analytical transfer function propose a methodology based on the theory of dynamic systems of an input and an output. That is, for any input $x(t)$, the output $y(t)$ is given by the convolution integral.

$$
f(t)=(g * q)(t)=\int_{-\infty}^{\infty} q(\tau) g(t-\tau) d \tau
$$

Mathematically, in terms of heat flow $q$ (input) and temperature $T$ (response), we have:

$$
\begin{aligned}
T_{1}(x, t) & =h_{1}(x, t) * q(t) \\
& =\int_{0}^{t} h_{1}(x, t-\tau) q(\tau) d \tau \\
T_{2}(x, t) & =h_{2}(x, t) * q(t) \\
& =\int_{0}^{t} h_{1}(x, t-\tau) q(\tau) d \tau
\end{aligned}
$$

where the function $h(x, t)=0$ for $\tau<0$.

Knowing the FG that characterizes the problem is possible to identify the system impulsive response, and therefore, a transfer function. The transfer 
function of a system is defined as the Laplace transform of the system's impulsive response.

Thus, since the FG of the problem $X 2 C 12$ whose solution is given by the Eq.(1) is known, and using the Convolution theorem given by Eq. (6) we have

$$
\begin{aligned}
& \Theta_{1}(x, t)=T_{1}(x, t) \\
& =\frac{\alpha_{1}}{k_{1}} \int_{0}^{t} G_{11}(x, t \mid 0, t-\tau) q(t) d \tau \\
& \Theta_{2}(x, t)=T_{2}(x, t) \\
& =\frac{\alpha_{1}}{k_{1}} \int_{0}^{t} G_{21}(x, t \mid 0, t-\tau) q(t) d \tau
\end{aligned}
$$

Substituting the GF that characterizes the $X 2 C 12$ problem andobserved that Eq.(7) is given by the product of terms that are dependent on $\tau$ and the delay $t-\tau$, which characterizes convolution theorem Eq.(5). Since the transfer function is independent of input and response, the Dirac delta function, $q(t)=\delta(t)$, is proposed as input signal (heat flux), so Eq. (6) become as follows:

$$
\begin{aligned}
T_{1}(x, t) & =h_{1}(x, t) * \delta(t) \\
& =\int_{0}^{t} h_{1}(x, t-\tau) \delta(\tau) d \tau \\
T_{2}(x, t) & =h_{2}(x, t) * \delta(t) \\
& =\int_{0}^{t} h_{1}(x, t-\tau) \delta(\tau) d \tau
\end{aligned}
$$

Note that from the neutral element property of the convolution, that $h * \delta=h$, thus, we get the impulsive response without solve the integral. Like this,

$$
\begin{aligned}
h_{1}(x, t)=\frac{\alpha_{1}}{k_{1}} \sum_{n=1}^{\infty} & \frac{1}{N_{x}} \cos (\gamma x) \\
& \times \int_{0}^{t} \delta(\tau) e^{-\lambda_{n}^{2}(t-\tau)} d \tau
\end{aligned}
$$

$$
\begin{aligned}
& h_{2}(x, t)_{\infty} \\
& =\frac{\alpha_{1}}{k_{1}} \sum_{n=1}^{\infty} \frac{1}{N_{x}}\{[\cos (\eta b) \cos (\gamma b) \\
& \left.+\left(\frac{k_{1}}{k_{2}}\right)\left(\frac{\gamma}{\eta}\right) \operatorname{sen}(\gamma b) \operatorname{sen}(\eta b)\right] \cos (\eta x) \\
& +[\cos (\gamma b) \operatorname{sen}(\eta b) \\
& \left.\left.-\left(\frac{k_{1}}{k_{2}}\right)\left(\frac{\gamma}{\eta}\right) \operatorname{sen}(\gamma b) \cos (\eta b)\right] \operatorname{sen}(\eta x)\right\} \\
& \times \int_{0}^{t} \delta(\tau) e^{-\lambda_{n}^{2}(t-\tau)} d \tau
\end{aligned}
$$

Given the transfer function of the problem $X 2 C 12$, by means of the Laplace transform of the impulse response, and as the knowledge of the system response (experimental or hypothetical temperature) is possible to obtain an estimate for the excitation of the system, it is the heat flux. This inverse proposed procedure will be presented next.

It is known that, for a linear dynamic system, the relation between input and output in the complex variable $s$ is given by the multiplication expressed in Eq. (10), or in the time domain by convolution.

$$
Y(s)=H(s) \cdot X(s)
$$

Thus, the analysis of dynamic systems is facilitated by the use of the Laplace transform because it provides the mathematical relationship between the input and output of the dynamic system. In terms of the heat/temperature couple pair.

$$
\begin{gathered}
\mathcal{L}\left[T_{2}(x, t)\right]=\mathcal{L}[h(x, t) * q(t)] \rightarrow T_{2}(x, s) \\
=H(x, s) \cdot q(s)
\end{gathered}
$$

Note that Eq. (11) is a multiplication, then we can rewrite as

$$
q(s)=\frac{1}{H(s, x)} T(x, s)
$$

Also, in the time domain, the inverse Laplace transform is applied, and the convolution is obtained, in mathematical terms is given by:

$$
\begin{aligned}
\mathcal{L}^{-1}[q(s)] & =\mathcal{L}^{-1}\left[\frac{1}{H(s, x) \cdot T(x, s)}\right] \\
& \Rightarrow q(t) \frac{1}{h(x, t)} * T(x, s)
\end{aligned}
$$

Numerically, this operation described by Eq. (13) will be performed using the MATLAB software by the following functions: fft and ifft which respectively apply the fast Fourier transform and the inverse transform to the functions $T, q$ and $h$.

\section{RESULTS AND DISCUSSION}

To obtain the solution of the one-dimensional direct problem, $X 2 C 12$, given by $\mathrm{Eq}$. (4), called a hybrid, it is necessary to consider a discrete heat flux, $q=\left[q_{1}, q_{2} \ldots q_{n}\right]$. In this way, we construct it in a vector form using MATLAB, as a triangular pulse, by means of the functiontripuls $\left(q=c_{1} * \operatorname{tripuls}(t-\right.$ $\left.\left.c_{2}, c_{2}\right)\right)$. The physical and geometric characteristics is, thermal conductivity, $k_{1}=401[\mathrm{~W} / \mathrm{mK}], \quad k_{2}=$ $401[\mathrm{~W} / \mathrm{mK}]$, thermal diffusivity, $\alpha_{1}=117 \times$ $10^{-6}\left[\mathrm{~m}^{2} / \mathrm{s}\right], \alpha_{2}=117 \times 10^{-6}\left[\mathrm{~m}^{2} / \mathrm{s}\right]$, temperature initial $T_{0}=0[\stackrel{o}{o}]$, plate length $L=10 \times 10^{-2}[\mathrm{~m}]$ and $\quad d t=1[s], t=[0: d t: t f], t f=1024[s]$, constants $c_{1}$ and $c_{2}$ can assume any value, among them were chosen: $c_{1}=3 \times 10^{5}$ and $c_{2}=300$. Fig. (3) shows the triangular heat flux. The temperature distribution is obtained for the positions $x=0, b$ 
and $L$ as shown in Fig. (4), in this way, by knowing the heat flow, the direct problem is established.

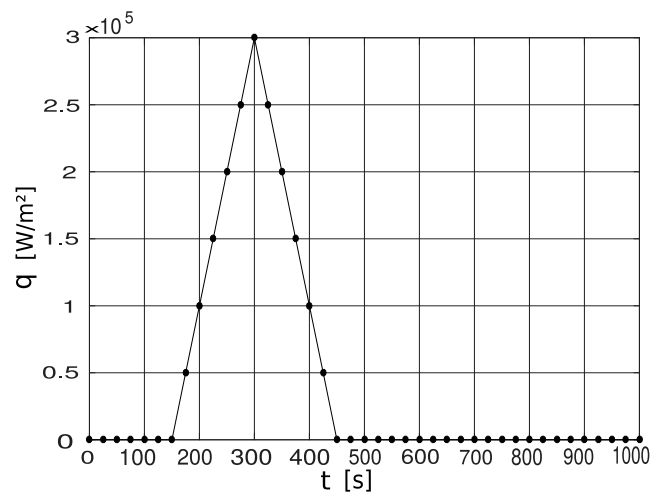

Figure 3. Triangular heat flux pulse that will be used to determine the thermal profile.

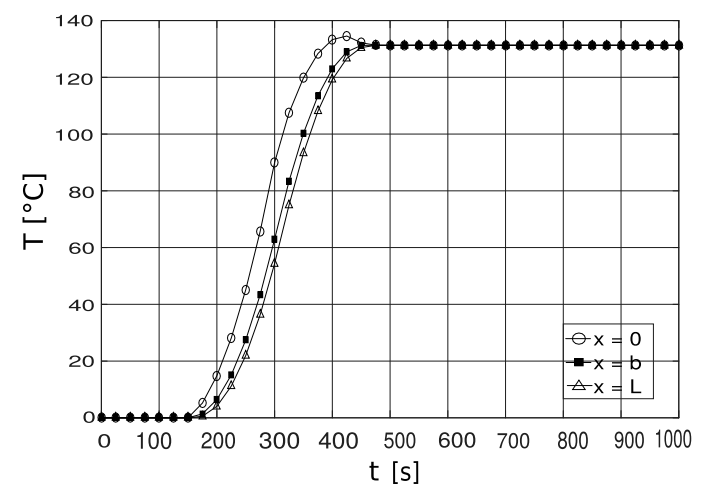

Figure 4. Temperature profile obtained using the triangular heat flux pulse.

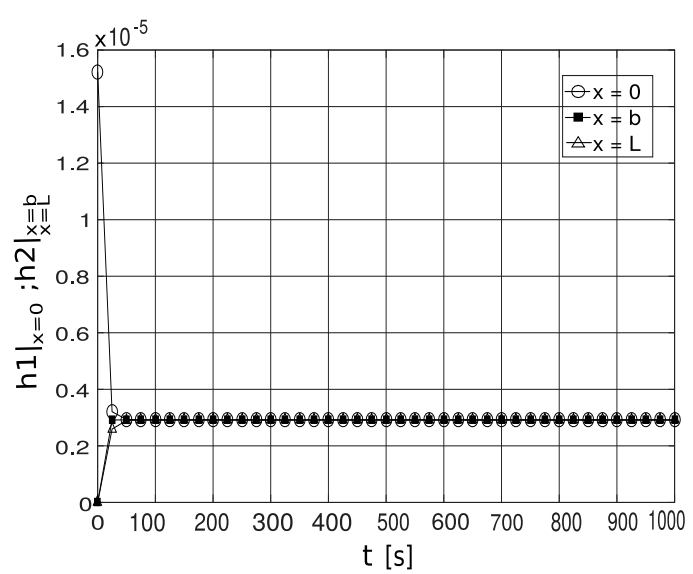

Figure 5. Impulsive response determined by impulsive response equation.

The impulsive response showed by Fig.(5) were calculated for $x=0, x=b$ and $x=L$ positions. We can note by Fig. (5) that, the impulsive response presents a decreasing behavior from the maximum value.

Thus, using the thermal profile and impulsive response data we can determine by Eq. (12) at frequency domain. To do this, were used the fft (Fast Fourier Transform) Matlab function.

In this sense, The Figs.(6-11) show the heat flux estimated and a comparison with simulated heat flux.

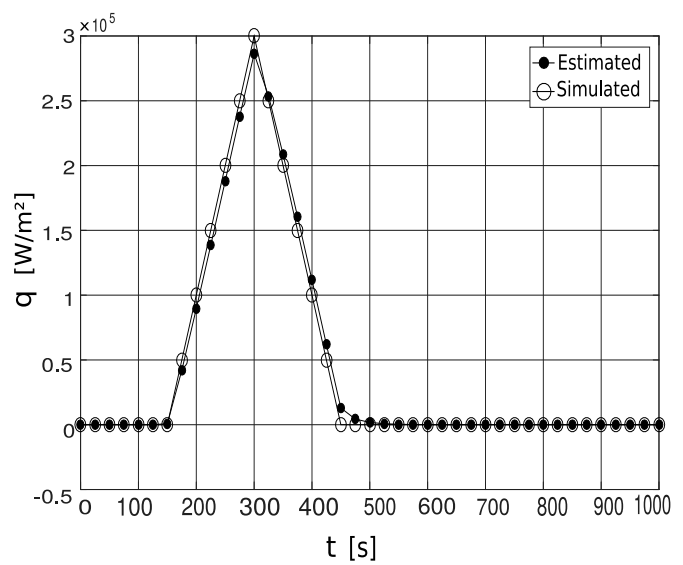

Figure 6. Estimated and simulated heat flux for $\boldsymbol{x}=\mathbf{0}$ position.

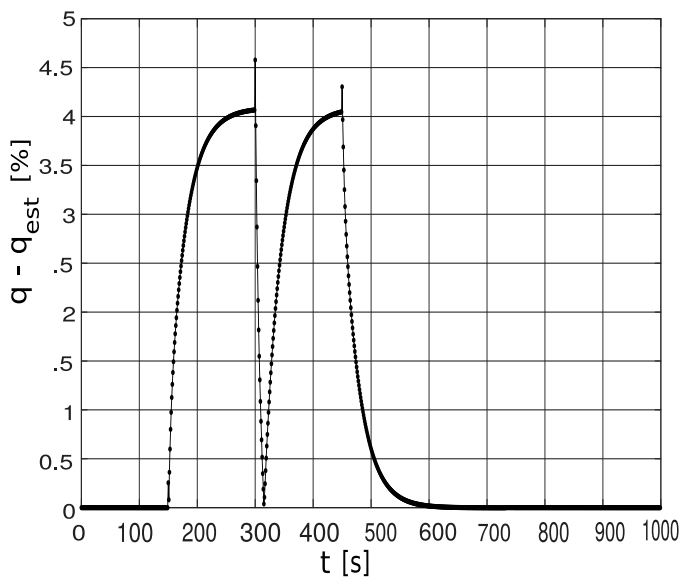

Figure 7. Relative difference between estimated and simulated heat flux for $\boldsymbol{x}=\mathbf{0}$ position.

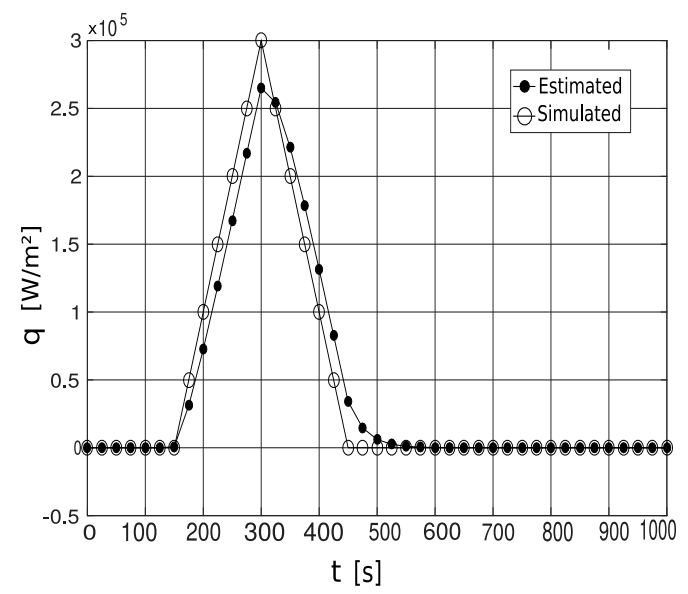

Figure 8.Estimated and simulated heat flux for $\boldsymbol{x}=\boldsymbol{b}$ position. 


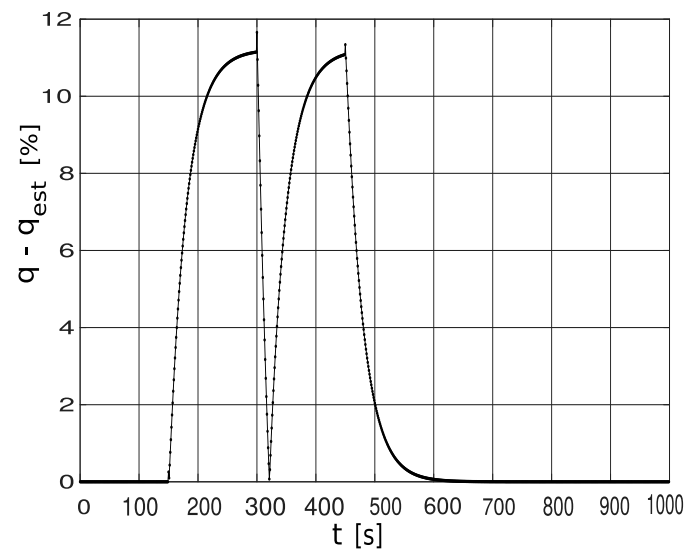

Figure 9.Relative difference between estimated and simulated heat flux for $\boldsymbol{x}=\boldsymbol{b}$ position.

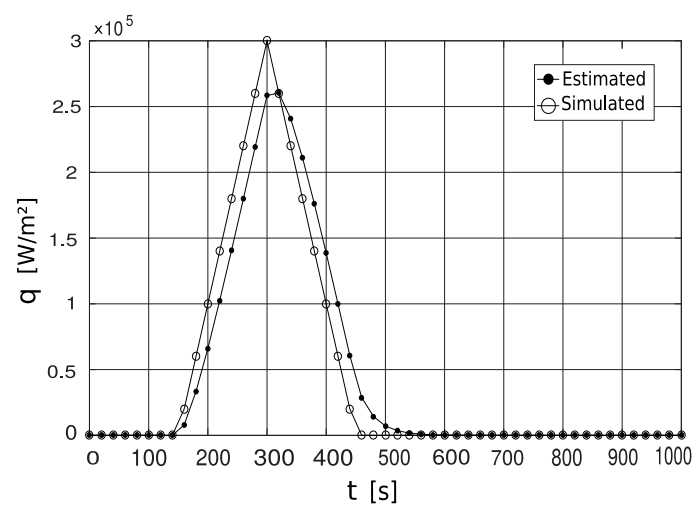

Figure 10.Estimated and simulated heat flux for $\boldsymbol{x}=\boldsymbol{L}$ position.

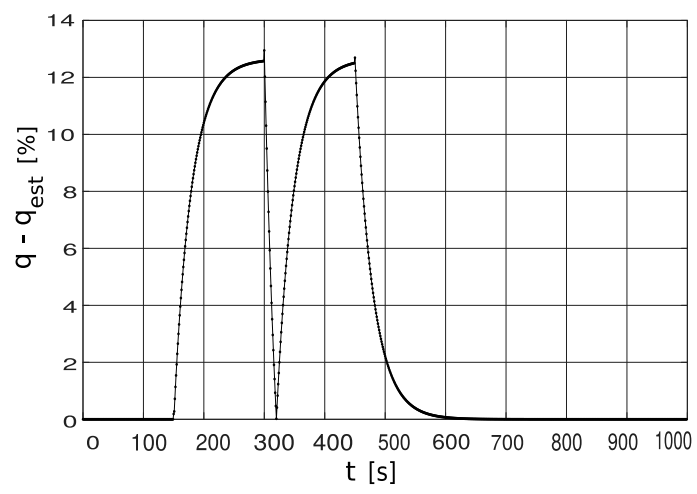

Figure 11.Relative difference between estimated and simulated heat flux for $\boldsymbol{x}=\boldsymbol{L}$ position.

\section{CONCLUSIONS}

This work was proposed a heat flux estimate method approach when we have a multilayer heat conduction problem. These kind of heat conduction problems present a mathematical difficulty due a model complexity and boundary condition combination.
In this sense, was showed an analytical method to solve the direct problem, was used the Green's function method that shown an excellent approach to handle of these mathematical complexity. Also, was proposed an adaptation of TFBGF method to determine a multilayer analytical transfer function.

Moreover, knowing the experimental (or theoretical) temperatures and the transfer function we can determine the heat flux doing a division of temperatures and transfer function at Laplace domain. In this way, the heat fluxes were estimated for $x=0, x=b$ and $x=L$ positions.

Thus, we can see in Figs. (6-8-10) the estimated heat flux compared to simulated heat flux, the relative differences showed were 4,5\%,11,7\% and $13 \%$ respectively to $x=0, x=b$ and $x=L$ positions Figs. (7-9-11). These behaviors were expected due the $x=0$ position is closer to heat flux position and the other positions are moving away from the heat source. Even so the method showed a good accuracy, also a great mathematical tool to solve this kind of heat problems.

\section{ACKNOWLEDGEMENTS}

The authors would like thank to the Brazilian agencies CAPES, FAPEMING and CNPq.

\section{REFERENCES}

Beck, J., 1992, Heat Conduction Using Green's Functions in Computational and Physical Processes in Mechanics and Thermal Sciences, Hemisphere Pub. Corp. ISBN 9781560320968.

Cole, K., Haji-Sheikh, A., Beck, J. V., and Litkouhi, B., 2010, Heat Conduction Using Green's Function, Series in Computational and Physical Processes in Mechanics and Thermal Sciences, Taylor \& Francis, ISBN 9781439813546.

Fernandes, A. P., 2009, Funções de Green: Soluções Analíticas Aplicadas a Problemas Inversos em Condução de Calor, Master Thesis. (in Portuguese).

Hahn, D., and Ozisik, N., 2012, Heat Conduction, Wiley, ISBN 9781118330111.

Haji-Sheik, A., and Beck, J. V., 2000, An Efficient Method of Computing Eigenvalues in Heat Conduction, Numerical Heat Transfer, Part B, Fundamentals, Vol. 38, No. 2, pp. 133-156.

Haji-Sheikh, A., 2014, Two-Layer Slab with Perfect Contact Between Layers; with Zero in Heat Flux at One Boundary, Zero Heat Flux at Other Boundary.

Haji-Sheikh, A., and Beck, J., 2002, Temperature Solution in Multi-dimensional MultiLayer Bodies, International Journal of Heat and Mass Transfer, Vol. 45, No. 9, pp. 1865-1877.

Oliveira, G. C., 2015, Solução Analítica Multicamada: aplicação em Ferramentas Revestidas. 
Master Thesis, Universidade Federal de Uberlândia. (in Portuguese)

Özisik, M. N., 1993, Heat Conduction, WileyInterscience Publication, Wiley, Nova Iorque. 